\title{
Validade das medidas do tempo sentado do questionário IPAQ-versão curta em universitários brasileiros
}

\author{
Validity of measure of sitting time of IPAQ Questionnaire short version in \\ undergraduate from Brazil
}

\section{AUTORES \\ Dayana Chaves Franco ${ }^{1}$ (D) \\ Gildeene Silva Farias² (D) \\ Andreia Pelegrini ${ }^{3}$ (iD) \\ Jair Sindra Virtuoso Junior ${ }^{4}$ (D) \\ Thiago Ferreira de Sousa ${ }^{5}$ (D) \\ 1 Centro Universitário de Maringá, Curso de \\ Educação Física, Maringá, Paraná, Brasil. \\ 2 Faculdade Estácio de Teresina, Curso de \\ Educação Física, Teresina, Piauí, Brasil. \\ 3 Universidade do Estado de Santa Catarina, \\ Centro de Ciências da Saúde e do Esporte, \\ Florianópolis, Santa Catarina, Brasil. \\ 4. Universidade Federal do Triângulo Mineiro, \\ Departamento de Ciências do Esporte, Uberaba, \\ Minas Gerais, Brasil. \\ 5 Universidade Federal do Recôncavo da Bahia, \\ Centro de Formação de Professores, Amargosa, \\ Bahia, Brasil.}

\section{CONTATO}

Dayana Chaves Franco

dayanafranco18@gmail.com

Rua Men de Sá, 1805. Vila Bosque, Maringá,

Paraná, Brasil.

CEP: 87005-010.

DOI

$10.12820 /$ rbafs. $26 \mathrm{e} 0223$

\section{(cc) BY}

Este trabalho está licenciado com uma Licença Creative Commons - Atribuição 4.0 Internacional.

\begin{abstract}
RESUMO
Os objetivos deste estudo foram estimar o nível de reprodutibilidade das medidas do tempo sentado do questionário International Physical Activity Questionnaire (IPAQ-versão curta) e analisar a validade concorrente dessas medidas em relação ao tempo sedentário mensurado pelo acelerômetro em universitários. Realizou-se um estudo correlacional com amostra de 61 universitários de uma instituição do ensino superior de Minas Gerais, Brasil, que autopreencheram as medidas do tempo sentado do IPAQ, intercalados por dois momentos diferentes para fins da análise de reprodutibilidade, e usaram o acelerômetro para estimativa do tempo sedentário e emprego como padrão ouro na análise de validade concorrente. Empregou-se as análises de correlação, via coeficiente de correlação intraclasse (CCI) e gráfico de dispersão de Bland-Altman, para analisar a concordância. O nível de significância adotado foi de 5\%. A média de idade foi de 21,54 anos e $62,3 \%$ foram do sexo feminino. A reprodutibilidade do tempo sentado durante um dia de semana foi moderada (CCI = 0,51; $\mathrm{p}<$ $0,01)$, enquanto em um dia do final de semana foi baixa $(\mathrm{CCI}=0,30 ; \mathrm{p}=0,01)$. As diferenças médias entre o tempo sentado pelo IPAQ e o tempo sedentário pelo acelerômetro, em um dia da semana foi de -51 minutos e em um dia do final de semana foi de $-64,7$ minutos. Conclui-se que houve correlações e concordâncias satisfatórias tanto na reprodutibilidade quanto na comparação com a medida critério (acelerômetro) das medidas do tempo sentado mensurado pelo IPAQ para aplicação em universitários.
\end{abstract}

Palavras-chave: Comportamento sedentário; Estudantes; Estudos de validação.

\section{ABSTRACT}

The objectives of this study were to estimate the level of reproducibility of the sitting time measurements of the International Physical Activity Questionnaire (IPAQ-short version), and to analyze the concurrent validity of these measurements in relation the sedentary time measured by the accelerometer, in university students. A correlational study was carried out with a sample of 61 university students of a bigher education institution from Minas Gerais, Brazil, who self-completed the IPAO sitting time measurements, with interval of seven days for the purposes of the reproducibility analysis and used the accelerometer to estimate sedentary time and employment as the gold standard in the analysis of concurrent validity. The correlation analyzes via intraclass correlation coefficient (ICC) and Bland-Altman scatter plot were used to analyze the agreement. The significance level adopted was 5\%. The average age was 21.54 years and $62.3 \%$ were female. The reproducibility of sitting time on a weekday was moderate $(I C C=0.51 ; p<0.01)$, while on a weekend day it was low $(I C C=0.30 ; p=0.01)$. The mean differences between sitting time using the IPAQ and sedentary time using the accelerometer on a weekday was -51 minutes and on a weekend was -64.7 minutes. Concluded that there were satisfactory correlations and agreements both in reproducibility and in comparison, with the criterion measure (accelerometer) of the sitting time measures measured by the IPAQ for application in university students.

Keywords: Sedentary behavior; Students; Validation studies.

\section{Introdução}

O comportamento sedentário (CS) é caracterizado como atividades realizadas na posição sentada, deitada ou reclinada, que possuem gasto energético próximo aos níveis de repouso ( $<1,5 \mathrm{MET}$ - equivalentes metabólicos $)^{1}$. Os estudos têm demonstrado que o elevado tempo dispendido nessas atividades está associado a fatores negativos para a saúde, como a obesidade ${ }^{2,3}$, depressão $\mathrm{O}^{2,4}$ e inclusive a mortalidade por todas as causas ${ }^{5}$.

As informações sobre este comportamento podem ser obtidas tanto de forma objetiva, com a utilização de aparelhos como o acelerômetro ${ }^{6}$, quanto de forma 
subjetiva, por meio de questionários ${ }^{7}$. A utilização de questionários para a mensuração do CS ainda representa uma das principais formas empregadas nos estudos epidemiológicos, conforme foi mostrado em revisão sistemática sobre o CS em adolescentes ${ }^{8}$ e universitários ${ }^{9}$. Pois os métodos indiretos de mensuração possuem baixo custo e facilitam a coleta de dados de um número elevado de participantes ${ }^{10}$.

Dessa forma, é de suma importância que essas medidas, obtidas de forma subjetiva, sejam confiáveis e apresentem índices psicométricos satisfatórios. Dentre as medidas do CS é possível citar como exemplo, o tempo sentado, que permite uma aproximação ao tempo global em $\mathrm{CS}^{11}$, que pode ser mensurado pelo acelerômetro ${ }^{6}$. O International Physical Activity Questionnaire (IPAQ) é um dos principais instrumentos para a mensuração do tempo sentado em diferentes grupos populacionais e foi validado, inclusive em brasileiros, para a aplicação em adolescentes ${ }^{12}$, adultos ${ }^{13-15}$ e idosos ${ }^{16}$.

$\mathrm{Na}$ pesquisa realizada com adolescentes brasileiros, as réplicas do tempo sentado apresentaram valores de correlação maiores que 0,58 em moças e rapazes ${ }^{12}$. Em estudos com adultos de diferentes países os índices de reprodutibilidade observados foram acima de 0,7 , com valores específicos para os brasileiros de $0,79^{13}$. Em relação a validação concorrente, em comparação ao acelerômetro Computer Science and Aplication's (CSA), as correlações em adultos variaram de 0,07 a 0,61, sendo os menores valores de concordância observados em brasileiros ${ }^{13}$.

Em estudo de validação do tempo sentado do questionário IPAQ em universitários colombianos, foi observado baixo nível de correlação (coeficiente de correlação intraclasse, CCI = 0,234; IC95\%: 0,011 $0,435)$ e subestimação dos minutos nesse comportamento quando comparados ao acelerômetro (diferença média de 19,5 minutos/dia, via gráfico de dispersão de Bland-Altman; variação do limite de confiança a $95 \%$ de $-339,4$ a 378,4 minutos) ${ }^{17}$. Por outro lado, torna-se essencial conhecer a qualidade do instrumento IPAQ para a aplicação em pesquisa com universitários brasileiros, haja vista a realização de estudos nesse grupo, para a mensuração tanto da atividade física ${ }^{18}$ quanto do tempo sentado 9 . Soma-se a tais exposições as especificidades do público universitário, quanto a amplitude das faixas de idade com vínculo com a universidade ${ }^{9} \mathrm{e}$ por conta da elevada demanda de tempo em atividades acadêmicas de estudos e socialização, normalmente realizadas na posição sentada, podendo estar associadas ao uso de computadores ${ }^{19}$, que aumentam com o ingresso na universidade ${ }^{20}$.

Considerando que o nível de saúde geral dos universitários necessita de atenção especial em pesquisas, em virtude do impacto na percepção de qualidade de vida $^{21}$, configura-se como relevante o conhecimento acerca das capacidades psicométricas dos instrumentos usados nesse grupo populacional e assim facilitar o desenvolvimento de políticas institucionais nas universidades com base em informações precisas. Este estudo teve como objetivos, estimar o nível de reprodutibilidade das medidas do tempo sentado do questionário IPAQ versão curta, e analisar a validade concorrente dessas medidas em relação ao tempo sedentário mensurado pelo acelerômetro, em universitários de uma instituição do ensino superior de Minas Gerais, Brasil.

\section{Métodos}

Este estudo correlacional, faz parte da etapa de validação das medidas do CS para aplicação em pesquisa com universitário, intitulada de "Perfil do estilo de vida e qualidade e vida dos estudantes da Universidade Federal do Triângulo Mineiro", aprovada pelo Comitê de Ética em Pesquisa (CEP) da Universidade Federal do Triângulo Mineiro (UFTM), sob número de parecer 2.402.734 de 2017 e seguiu a Resolução no 466/12, do Conselho Nacional de Saúde (CNS). Os universitários participantes assinaram o termo de consentimento livre e esclarecido, previamente à participação voluntária na pesquisa.

A população alvo compreendeu os universitários dos cursos de graduação da UFTM, Minas Gerais, Brasil. Considerou-se como critério de inclusão, ter idade igual ou superior a 18 anos e estar com matrícula ativa, comprovada por meio de crachá da instituição. Foram considerados como critérios de exclusão pertencer a cursos de ensino a distância, do ensino técnico, ter matrícula especial como ser portador de diploma do ensino superior e não pertencer ao campus da cidade de Uberaba, Minas Gerais. A amostra estimada para a realização da análise de validade concorrente foi calculada, a priori, no software Bioestat (versão 5.3), e considerado o coeficiente de correlação de $0,50^{22}$, poder de $80 \%$, nível de confiança de $95 \%$, perfazendo amostra mínima de 30 universitários. Os participantes deste estudo foram selecionados por conveniência e considerando que esta etapa ocorreu de forma simultânea ao inquérito principal, os voluntários não fizeram parte da pesquisa principal.

As coletas de dados foram realizadas entre os meses 
de abril a novembro de 2018. Os universitários foram abordados nas dependências da universidade, antes ou após as aulas, e foram convidados a participar da pesquisa, que foi realizada mediante o autopreenchimento de um questionário com 19 perguntas fechadas, em dois momentos, e fizeram uso de acelerômetro. $\mathrm{O}$ instrumento foi respondido em sala de aula, de forma individual ou em pequenos grupos com no máximo 10 universitários e assistidos por um aplicador. $\mathrm{O}$ tempo médio de preenchimento do questionário composto por perguntas sociodemográficas, de vínculo com a universidade, tempo de sono, comportamentos de tela e de realização de pausas no $\mathrm{CS}$, mais as duas perguntas do tempo sentado do questionário IPAQ versão cur$\mathrm{ta}^{15}$, foi de 15 minutos.

Após ter respondido o questionário na primeira etapa (teste), este foi devolvido ao pesquisador, sendo entregue o acelerômetro, da marca ActiGraph GT3X, com as devidas orientações de uso, que foram: usar o aparelho na cintura ${ }^{23}$, preso a um cinto elástico, do lado direito do corpo, que utilizasse durante o dia todo, por pelo menos sete dias da semana consecutivos, incluindo dois dias de final de semana e apenas retirassem o aparelho para realização de atividades aquáticas, no banho e para dormir. Após decorrido o período de utilização do equipamento (sete dias), no ato da entrega, os universitários responderam novamente o questionário (reteste).

A variável padrão ouro deste estudo foi o acelerômetro. Considerou-se como válidas as informações dos acelerômetros que apresentaram a quantidade mínima de 4 dias de uso, sendo pelo menos 1 dia de final de semana, com pelo menos 10 horas por dia de tempo de utilização. Os dados foram coletados com uma taxa de amostragem de $80 \mathrm{~Hz}$, agregados para períodos de dois minutos. Em relação ao acelerômetro, definiu-se como ponto de corte do tempo sedentário $<100$ counts $^{24}$. Os dados dos acelerômetros foram baixados no software ActiLife v6 13.3, e os dados foram analisados em minutos do tempo sedentário como tempo médio em um dia da semana e em um dia do final de semana, obtidos por meio da soma dos minutos de cada dia, divididos pela quantidade de dias de uso.

As variáveis testadas em relação a medida critério foram as informações do tempo sentado em minutos em um dia da semana e um dia do final de semana, estimados pelo IPAQ. As horas foram transformadas em minutos. As seguintes questões compreendem as medidas do tempo sentado: "Quanto tempo no total você gasta sentado durante um dia de semana?" e "Quanto tempo no total você gasta sentado durante um dia do final de semana?". Os voluntários deveriam recordar em um dia típico o tempo em horas e minutos que permanecem sentados no trabalho, na escola ou faculdade, em casa e durante o tempo livre, como por exemplo, estudando, ou em descanso, lendo ou assistindo televisão, não sendo considerado o tempo gasto sentado no ônibus, trem, metrô ou carro.

Optou-se pela aplicação apenas das perguntas do tempo sentado, sem as perguntas sobre atividade física mensuradas pelo IPAQ pois não compreendiam o escopo do estudo e para evitar o aumento do tempo de preenchimento e assim contribuir com a qualidade das informações. Para manter a qualidade dos dados, os valores discrepantes foram corrigidos de acordo com a informação do tempo de sono para um dia da semana e um dia do final de semana ${ }^{25}$.

As variáveis para a caracterização da amostra foram: sexo (masculino e feminino), idade em anos completos (variável quantitativa), área de estudo, categorizada de acordo com a área de conhecimento com base a informação do curso, dicotomizada em Ciências da Saúde (Educação Física, Enfermagem, Fisioterapia, Nutrição e Terapia ocupacional) e Outras (Engenharia ambiental, Engenharia civil, Engenharia de alimentos, Engenharia mecânica, Engenharia química, Física, História, Letras, Matemática, Química, Psicologia e Serviço so(ial ${ }^{26}$ e situação conjugal (com parceiro e sem parceiro).

Os dados foram tabulados no software Excel, versão 2007. As análises estatísticas foram realizadas no software IBM Statistics SPSS versão 24. Foram realizados os testes de simetria e curtose para a estimativa de normalidade dos dados, considerando aceitáveis os valores maiores que -2 e até 2 . As descrições das informações foram realizadas via média, desvio padrão (DP), valores mínimo e máximo.

Os processos adotados para a análise da reprodutibilidade foram: a) comparações do tempo sentado, estimados pelas perguntas do questionário IPAQ $\mathrm{em}$ minutos, entre as aplicações (teste e reteste) via teste $t$ de Student para amostras pareadas; b) CCI para estimar o índice de reprodutibilidade entre as medidas das duas aplicações das perguntas do questionário IPAQ; c) nível de concordância do tempo sentado em minutos entre as aplicações das medidas, via gráficos de dispersão de Bland-Altman ${ }^{27}$, realizados no software MedCalc (versão 9.1.0.1).

Os processos adotados para a análise da validade concorrente foram: a) comparações entre o tempo sen- 
tado, mensurado na primeira aplicação das perguntas do questionário IPAQ e o tempo sedentário via acelerômetro, ambos em minutos, via teste $t$ de Student para amostras pareadas; b) CCI para estimar o nível de validade concorrente entre o tempo sentado na primeira aplicação das perguntas do questionário IPAQ com o tempo sedentário obtido pelo acelerômetro; c) nível de concordância entre o tempo sentado em minutos, referente a primeira aplicação e o tempo sedentário mensurado pelo acelerômetro, por meio dos gráficos de dispersão de Bland-Altman ${ }^{27}$, elaborados no software MedCalc (versão 9.1.0.1).

A classificação empregada para o CCI foi de 0,90 a 1,00: muito alta; de 0,70 a 0,90: alta; de 0,50 a 0,70: moderada; de 0,30 a 0,50: baixa e de 0 a 0,30: correlação insignificante ${ }^{28}$. $\mathrm{O}$ nível de significância adotado foi de $5 \%$.

\section{Resultados}

Participaram do estudo 61 universitários na primeira aplicação do questionário. Na Tabela 1 são apresentadas as características da amostra. Entre os participantes na primeira aplicação, a média de idade foi de 21,54 anos, com a maior parte composta por universitários do sexo feminino $(62,3 \%)$, da área da saúde $(42,6 \%)$ e sem companheiro $(95,1 \%)$. Porém, no reteste houve a participação de 57 universitários, e assim quatro universitários não compareceram no prazo de sete dias para a participação da segunda aplicação do questionário e devolução do acelerômetro. Destes que compareceram no segundo momento, 33 utilizaram o acelerômetro com informações que atendiam os critérios previamente definidos. Embora a menor quantidade de universitários com informações do acelerômetro, essas características não diferem do perfil de participantes da primeira aplicação do instrumento.

A Tabela 2 apresenta a análise descritiva do tempo sentado estimado pelo IPAQ e o tempo sedentário mensurado pelo acelerômetro. A média de exposição ao tempo sedentário durante um dia da semana foi de 595,40 minutos e em um dia do final de semana foi de 584,48 minutos. Durante um dia da semana, a média do tempo sentado foi de 528,69 minutos no teste, e no reteste foi de 500,53 minutos. Para um dia do fim de semana a média de tempo sentado foi de 520,82 minutos no teste, e no reteste foi de 513,68 minutos.

Tabela 1 - Características descritivas da amostra no teste, reteste e com dados do acelerômetro. Uberaba, Minas Gerais. 2018.

\begin{tabular}{|c|c|c|c|c|c|c|c|c|c|}
\hline \multirow[b]{2}{*}{ Variáveis } & \multicolumn{3}{|c|}{ Teste } & \multicolumn{3}{|c|}{ Reteste } & \multicolumn{3}{|c|}{ Com dados do acelerômetro } \\
\hline & $\mathrm{n}$ & $\begin{array}{c}\bar{x}(\mathrm{DP}) \\
\text { Mín-Máx }\end{array}$ & $\%$ & $\mathrm{n}$ & $\begin{array}{c}\bar{x}(\mathrm{DP}) \\
\text { Mín-Máx }\end{array}$ & $\%$ & $\mathrm{n}$ & $\begin{array}{c}\bar{x}(\mathrm{DP}) \\
\text { Mín-Máx }\end{array}$ & $\%$ \\
\hline \multicolumn{10}{|l|}{ Sexo } \\
\hline Masculino & 23 & - & 37,7 & 23 & - & 40,4 & 13 & - & 39,4 \\
\hline Feminino & 38 & - & 62,3 & 34 & - & 59,6 & 20 & - & 60,6 \\
\hline Idade em anos completos & 61 & $\begin{array}{c}21,54(3,5) \\
18-34\end{array}$ & - & 57 & $\begin{array}{c}21,46(3,3) \\
18-34\end{array}$ & - & 33 & $\begin{array}{c}21,52(3,3) \\
18-34\end{array}$ & - \\
\hline \multicolumn{10}{|l|}{ Área de estudo } \\
\hline Saúde & 26 & - & 42,6 & 24 & - & 42,1 & 19 & - & 57,6 \\
\hline Outras & 35 & - & 57,4 & 33 & - & 57,9 & 14 & - & 42,4 \\
\hline \multicolumn{10}{|l|}{ Situação conjugal } \\
\hline Sem parceiro & 58 & - & 95,1 & 55 & - & 96,5 & 32 & - & 97,0 \\
\hline Com parceiro & 03 & - & 4,9 & 02 & - & 3,5 & 01 & - & 3,0 \\
\hline
\end{tabular}

$\overline{\mathrm{X}}=$ média; DP = desvio padrão; Mín. = Mínimo; Máx. = Máximo; \% = Proporção

Tabela 2 - Análise descritiva do tempo sentado estimado pelo questionário IPAQe tempo sedentário estimado pelo acelerômetro. Uberaba, Minas Gerais. 2018.

\begin{tabular}{lccccc}
\hline Variáveis & $\mathrm{n}$ & $\overline{\mathrm{x}}$ (DP); IC95\% & Min-Max & Sim. & Curt. \\
\hline Tempo sentado (minutos/1dia/semana) (teste) & 61 & $528,69(196,12) ; 478,46-578,92$ & $90-1.110$ & 0,242 & 0,462 \\
Tempo sentado (minutos/1dia/semana) (reteste) & 57 & $500,53(215,97) ; 443,22-557,83$ & $180-1.200$ & 0,928 & 0,640 \\
Tempo sentado (minutos/1dia/FS) (teste) & 61 & $520,82(207,05) ; 467,79-573,85$ & $60-1.200$ & 0,467 & 1,281 \\
Tempo sentado (minutos/1dia/FS) (reteste) & 57 & $513,68(193,43) ; 462,36-565,01$ & $180-1.200$ & 0,730 & 1,709 \\
Tempo sedentário (minutos/1dia/semana) & 33 & $595,40(183,00) ; 530,51-660,29$ & $192-1.130$ & 0,836 & 2,421 \\
Tempo sedentário (minutos/1dia/FS) & 33 & $584,48(172,86) ; 523,19-645,78$ & $151-976$ & $-0,168$ & 0,738 \\
\hline
\end{tabular}

$\overline{\mathrm{X}}(\mathrm{DP})$ = Média (desvio padrão); Mín. = Mínimo; Máx. = Máximo; Sim. = Simetria Curt. = Curtose; FS = Final de semana; IC95\% = Intervalo de Confiança a 95\%. 
Em relação a reprodutibilidade, houve correlação de baixa a moderada entre o tempo sentado durante um dia da semana $(\mathrm{CCI}=0,51 ; \mathrm{p}<0,01)$ e correlação baixa em um dia do final de semana $(\mathrm{CCI}=0,30 ; \mathrm{p}=$ $0,01)$, entre o teste e reteste das medidas do questionário IPAQ. Foram observadas médias iguais dos minu-
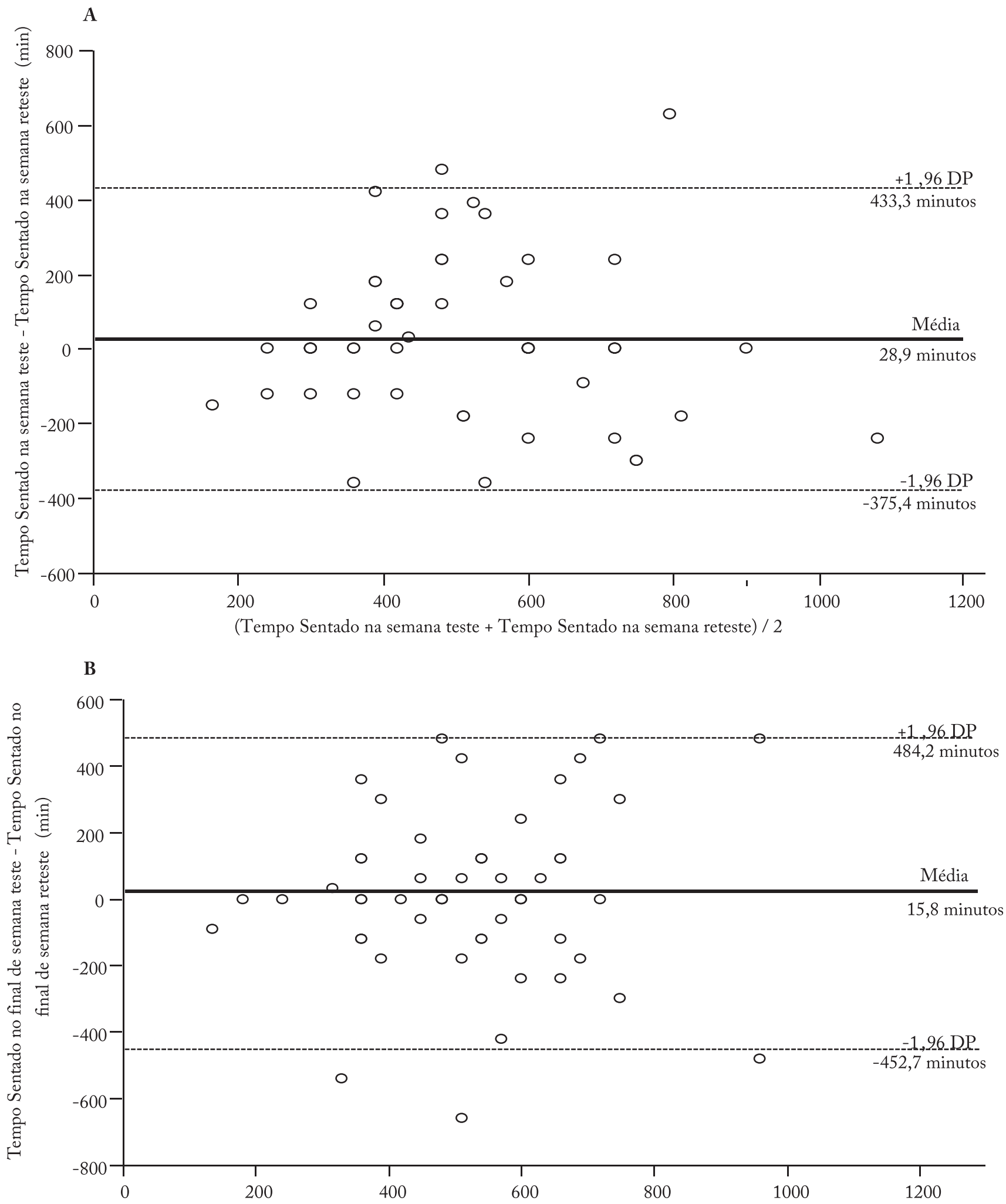

(Tempo Sentado no final de semana teste + Tempo Sentado no final de semana reteste) $/ 2$

Figura 1 - Gráfico de dispersão de Bland-Altman entre o tempo sentado no teste e reteste, em um dia da semana (Figura a) e um dia do final de semana (Figura b), ambos estimados pelo IPAQ. Uberaba, Minas Gerais. 2018. 
tos de tempo sentado em um dia da semana $(p=0,29)$ e um dia do final de semana $(\mathrm{p}=0,65)$. Os níveis de concordância entre o teste e reteste do tempo sentado são apresentados na Figura 1. Observou-se diferenças médias, entre o teste e reteste, de 28,9 minutos para um dia de semana e 15,8 minutos para um dia do final de semana.

Quanto a validade concorrente entre o tempo sentado e o tempo sedentário (Tabela 3), foi possível observar correlação baixa durante a semana $(\mathrm{CCI}=0,31)$ e baixa a moderada para o fim de semana $(\mathrm{CCI}=0,53)$. Observou-se médias estatisticamente iguais entre o tempo sentado e o tempo sedentário em relação a um dia da semana $(p=0,24)$ e um dia do final de semana $(\mathrm{p}=0,06)$. Sobre a concordância (Figura 2$)$, as diferenças médias entre o tempo sentado pelo IPAQ e tempo sedentário pelo acelerômetro, em um dia da semana foi de -51 minutos com variação da concordância de $-527,5(-1,96 \mathrm{DP})$ e 425,5 (+1,96 DP) minutos, e em um dia do final de semana foi de $-64,7$ minutos com variação da concordância de $-429,3(-1,96$ DP) e 300,0 $(+1,96 \mathrm{DP})$ minutos.

Tabela 3 - Correlações e comparação entre o tempo sentado estimado pelo IPAQ e tempo sedentário estimado pelo acelerômetro. Uberaba, Minas Gerais. 2018.

\begin{tabular}{lcccc}
\hline Variáveis & $\mathrm{n}$ & $\mathrm{CCI}$ & $\mathrm{p}^{*}$ & $\mathrm{p}^{* *}$ \\
\hline Tempo sentado (minutos/1dia/semana) (teste) & 33 & 0,31 & 0,04 & 0,24 \\
Tempo sedentário (minutos/1dia/semana) & & & & \\
$\begin{array}{l}\text { Tempo sentado (minutos/1dia/FS) (teste) } \\
\text { Tempo sedentário (minutos/1dia/FS) }\end{array}$ & 33 & 0,53 & $<0,01$ & 0,06 \\
\hline
\end{tabular}

$\mathrm{CCI}=$ Coeficiente de Correlação Intraclasse; ${ }^{*}$ p valor do CCI; ${ }^{* *} \mathrm{p}$ valor do teste $\mathrm{t}$ de student; FS = Final de semana.

\section{Discussão}

Neste estudo, as medidas do tempo sentado apresentaram em relação a reprodutibilidade, correlação baixa a moderada para o dia da semana e correlação baixa para o dia do final de semana, e a concordância entre o teste e reteste foi satisfatória. Sobre a comparação com a medida critério, o tempo sentado mensurado pelo IPAQ em sua versão curta apresentou correlação baixa para a informação do dia da semana e baixa a moderada para o dia do final de semana, com valores de concordância satisfatórios.

Os valores de correlação entre as duas aplicações das medidas do tempo sentado foram de CCI: 0,51 e CCI: 0,30, para um dia da semana e um dia do final de semana, respectivamente, com valores de médias esta- tisticamente iguais. Esses valores foram menores que o nível de correlação observada em estudo de validação com universitários colombianos, que observou CCI de 0,716 (IC95\%: 0,583-0,811) para o tempo senta$\mathrm{do}^{17}$. No estudo de validação inicial do IPAQ versão curta, realizado em diferentes países, os resultados das correlações foram maiores que esta investigação, com a variação de 0,58 em japoneses a 0,94 em finlandeses e estados unidenses ${ }^{13}$. Nesse mesmo estudo, a correlação, em adultos brasileiros foi de $0,79^{13}$. Embora os índices de correlação deste estudo sejam menores, quando comparado ao estudo de validação inicial do IPAQ $^{13}$ e outros estudos com adultos ${ }^{29}$ em diferentes países, os resultados desta pesquisa são considerados satisfatórios, por representarem medidas do tipo papel e caneta, que necessitam recordar o comportamento, além disso, apresentam baixo custo e são viáveis para o emprego em estudos de abrangência populacional, como em inquéritos com universitários.

Notou-se concordância entre as aplicações das medidas do tempo sentado (gráfico de dispersão de Bland-Altman) e médias estatisticamente iguais entre as réplicas das aplicações. As diferenças médias foram positivas entre as aplicações, o que configura a superestimação na primeira aplicação, porém, essas diferenças foram de 28,9 minutos e 15,8 minutos, em relação a um dia de semana e um dia de final de semana, respectivamente. Esses resultados convergem com as informações de reprodutibilidade do tempo sentado do IPAQ em jovens, tanto em moças (12 a 14 anos: 30 minutos para a semana; 15 a 18 anos: 32 minutos para o final de semana) quanto em rapazes (12 a 14 anos: 58 minutos para a semana; 15 a 18 anos: 16 minutos para o final de semana) brasileiros ${ }^{12}$, grupo que apresenta perfil de estudos que se assemelha aos universitários, especificamente, quanto a demanda de tempo para as atividades de aprendizagem.

Corroborando com as informações apresentadas na etapa de reprodutibilidade, observou-se concordância satisfatória entre as medidas do tempo sentado em relação ao tempo sedentário pelo acelerômetro com valores dentro dos limites aceitáveis de 95\% de confiança, especialmente para o tempo do final de semana, com melhor distribuição das diferenças médias. Por outro lado, o tempo referente ao dia da semana apresentou menor subestimação pelos universitários. Em estudo com universitários colombianos, notou-se menores diferenças médias que este estudo (19,5 minutos), mas, de forma semelhante houve variação ampla dos valo- 
res dentro do intervalo de $95 \%$ de confiança $(-339,4$ a 378,4 minutos), o que demonstra a característica de variabilidade das informações a esse comportamento nesse grupo.
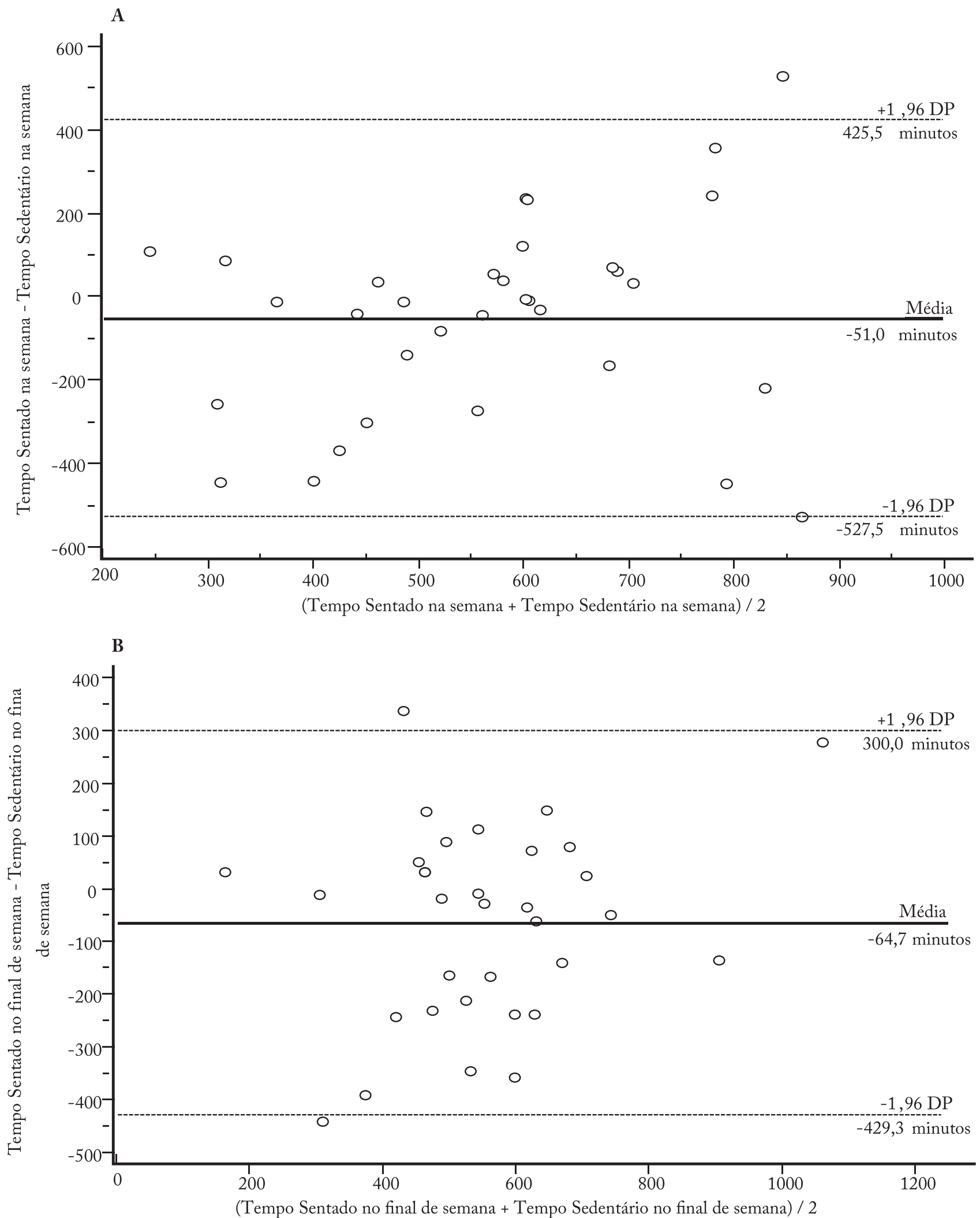

Figura 2 - Gráfico de dispersão de Bland-Altman entre o tempo sentado (teste) estimado pelo IPAQe tempo sedentário estimado pelo acelerômetro, por dia da semana (Figura a) e por dia do final de semana (Figura b). Uberaba, Minas Gerais. 2018. 
Associado a esses achados, observou-se correlação baixa para o dia da semana e baixa a moderada para o dia do final de semana. Estes resultados convergem com os níveis de validade desta medida em relação aos universitários colombianos ${ }^{17}$ e informações de diferentes países em relação a versão curta do instrumento em adultos, em comparação ao acelerômetro ${ }^{29}$. Além disso, nota-se que a presente medida demonstra melhor capacidade comparativa com a medida critério do que outras medidas do CS, como o tempo de tela (televisão, computador e vídeo-game) para a aplicação em universitários ${ }^{30}$.

Os menores valores de validade do tempo sentado em comparação as medidas diretas são consistentes, conforme observado em estudo de revisão sobre o tema ${ }^{22}$, o que demonstra a possibilidade de vieses de resposta, especialmente pela dificuldade de reportar com maior certeza a permanência em determinadas condutas ao longo do dia, conforme também observado em universitários colombianos ${ }^{17}$. Haja vista que medidas diretas, como o acelerômetro são sensíveis para a detecção dos breaks (pausas) entre os CS, bem como melhor caracterizar o tempo correto em atividades de menor gasto energético, como sentado, deitado e reclinado ${ }^{6,11}$. Por outro lado, dentre as medidas relativas ao CS, que podem mensurar tais informações em diferentes domínios ${ }^{9}$, o tempo sentado, estimado pelo IPAQ pode ser uma alternativa importante para comparação entre os estudos, como em grupos de universitários e assim evitar o uso de medidas elaboradas sem validação prévia ${ }^{9}$.

Este estudo apresenta limitações, como o método de seleção da amostra por conveniência, que pode favorecer a participação de universitários com maior autoeficácia em relação a comportamentos saudáveis, especialmente aqueles provenientes dos cursos da área das Ciências da Saúde, porém, de forma minimizar esse viés, recorreu-se a realização do convite à participação na pesquisa aos universitários de diferentes cursos da instituição. Análises em separado para homens e mulheres não foram realizadas neste estudo o que possivelmente pode representar uma limitação em virtude do tamanho amostral em cada grupo, no entanto, observou-se que as médias do tempo sentado na semana, final de semana e para o tempo sedentário neste estudo não divergiram entre os sexos (dados não mostrados), e assim permite caracterizar a relevância das análises de modo geral. Em contrapartida, esta pesquisa apresenta de forma positiva a utilização do acelerômetro como medida critério para a medida do tempo sedentário, uma vez que representa forma precisa de mensuração dessa conduta relacionada a saúde.

Conclui-se que as perguntas do tempo sentado do IPAQ apresentaram tanto para a reprodutibilidade quanto para a validade concorrente, níveis satisfatórios de índices psicométricos e assim podem ser utilizadas na população de universitários. Diante disso, para o levantamento das informações sobre esse tema, o emprego dessas medidas poderá ampliar o escopo do estado da arte sobre CS, bem como favorecem a comparação entre os estudos, de modo a auxiliar na implementação de políticas institucionais direcionadas a redução desse comportamento.

\section{Conflito de interesse}

Os autores declaram não haver conflito de interesse.

\section{Financiamento}

O presente trabalho foi realizado com apoio da Coordenação de Aperfeiçoamento de Pessoal de Nível Superior - Brasil (CAPES).

\section{Contribuição dos autores}

Franco DC realizou a coleta de dados, análise dos dados e escrita do artigo, que compõe a dissertação de mestrado intitulada "Comportamento sedentário em universitários: estimativas de acurácia, prevalências e fatores associados”; Farias GS realizou a coleta de dados e auxiliou na revisão crítica do artigo; Pelegrini A realizou a análise crítica do artigo; Virtuoso Junior JS realizou a análise crítica do artigo; Sousa TF realizou a orientação do artigo, auxiliou nas análises dos dados e revisão crítica do artigo.

\section{Referências}

1. Dunstan DW, Dogra S, Carter SE, Owen N. Sit less and move more for cardiovascular health: emerging insights and opportunities. Nat Rev Cardiol [journal on the internet]. 2021 [cited 2021 Jul 15]. Available from: https://doi.org/10.1038/ s41569-021-00547-y

2. Saunders TJ, McIsaac T, Douillette K, Gaulton N, Hunter S, Rhodes RE et al. Sedentary behaviour and health in adults: an overview of systematic reviews. Appl Physiol Nutr Metab. 2020;45(10):S197-S217.

3. Martínez-Ramos E, Beltran AM, Martín-Borràs C, Lasaosa-Medina L, Real J, Trujillo JM et al. Patterns of sedentary behavior in overweight and moderately obese users of the Catalan primary-health care system. PLoS ONE. 2018;13(1):e0190750.

4. Pozo-Cruz B, Alfonso-Rosa RM, McGregor D, Chastin SF, Palarea-Albaladejo J, del Pozo-Cruz J. Sedentary behaviour is associated with depression symptoms: compositional data analysis from a representative sample of 3233 US adults and older adults assessed with accelerometers. J Affect Disord. 2020;265(6):59-62. 
5. Ku PW, Steptoe A, Liao Y, Hsueh MC, Chen LJ. A cutoff of daily sedentary time and all-cause mortality in adults: a meta-regression analysis involving more than 1 million participants. BMC Med. 2018;16(74):16-74.

6. Meneguci J, Santos DAT, Silva RB, Santos RG, Sasaki JE, Tribess S, et al. Comportamento sedentário: conceito, implicações fisiológicas e os procedimentos de avaliação. Motri. 2015;11(1):160-74.

7. Prince SA, Melvin A, Roberts KC, Butler GP, Thompson W. Sedentary behaviour surveillance in Canada: trends, challenges and lessons learned. Int J Behav Nutr Phys Act. 2020;17(1):34.

8. Silva Filho RCS, Lemes TMMA, Sasaki JE, Gordia AP, Andaki ACR. Comportamento sedentário em adolescentes brasileiros: uma revisão sistemática. Rev Bras Ativ Fís Saúde. 2020;25:e0139.

9. Franco DC, Ferraz NL, Sousa TF. Comportamento sedentário em universitários: uma revisão sistemática. Rev Bras Cineantropom Hum. 2019;21:e56485.

10. Warren LM, Ekelund U, Besson H, Mezzani A, Geladas N, Vanhees L. Assessment of physical activity - a review of methodologies with reference to epidemiological research: a report of the exercise physiology section of the European Association of Cardiovascular Prevention and Rehabilitation. Eur J Cardiovasc Prev Rehabil. 2010;17(2):127-39.

11. Tremblay MS, Aubert S, Barnes JD, Saunders TJ, Carson V, Latimer-Cheung AE, et al. Terminology Consensus Project Participants. Sedentary behavior research network (SBRN) Terminology Consensus Project process and outcome. Int J Behav Nutr Phys Act. 2017;14(1):75.

12. Guedes DP, Lopes CC; Guedes JERP. Reprodutibilidade e validade do Questionário Internacional de Atividade Física em adolescentes. Rev Bras Med Esporte. 2005;36(1):79-97.

13. Craig CL, Marshall AL, Sjostrom M, Bauman AE, Booth ML, Ainsworth BE et al. International physical activity questionnaire: 12-country reliability and vality. Med Sci Sports Exerc. 2003;35(8):1381-5.

14. Hallal PC, Victora CG. Reliability and validity of the International Physical Activity Questionnaire (IPAQ). Med Sci Sports Exerc. 2004;36(3):556.

15. Matsudo S, Araújo T, Matsudo V, Andrade D, Andrade E, Oliveira LC, et al. Questionário internacional de atividade física (IPAQ): estudo de validade e reprodutibilidade no Brasil. Rev Bras de Ativ Fis Saúde. 2001;6(2):5-18.

16. Benedetti TB, Antunes PC, Rodrigues-Añez CR, Mazo GZ, Petroski EL. Reprodutibilidade e validade do Questionário Internacional de Atividade Física (IPAQ) em homens idosos. Rev Bras Med Esporte. 2007;13(1):11-6.

17. Arango-Vélez EF, Echavarría-Rodríguez AM, AguilarGonzález FA, Patiño-Villada FA. Validación de dos cuestionarios para evaluar el nivel de actividad física $\mathrm{y}$ el tiempo sedentario en una comunidad universitaria de Colombia. Rev Salud Pública. 2020;38(1):e334156.
18. Sousa TF. Inatividade física em universitários brasileiros: uma revisão sistemática. Rev Bras Ciênc Saúde. 2011;9(29):47-55.

19. Lourenço CLM, Sousa TF, Fonseca SA, Virtuoso Junior JS, Barbosa AR. Comportamento sedentário em estudantes universitários. Rev Bras Ativ Fís Saúde. 2016;21(1):67-77.

20. Prat IA, Viñolas EC, Cañas JCM, Wasley DA, PuigRibera A. From secondary school to university: associations between sport participation and total and domain-specific sedentary behaviours in Spanish students. Eur J Pediatr. 2020:179(10):1635-45.

21. Tavares CC, Cornélio, MPM; Nardelli GG, Gaudenci EM, Haas VJ, Pedrosa LAK. Saúde e qualidade de vida de universitários da área da saúde. Rev Fam Ciclos Vida Saúde Contexto Soc. 2019;7(1):53-64.

22. Healy GN, Clark BK, Winkler EA, Gardiner PA, Brown WJ, Matthews CE. Measurement of Adults' Sedentary Time in Population-Based Studies. Am J Prev Med. 2011;41(2):216-27.

23. Rosenberger ME, Haskell WL, Albinali F, Mota S, Nawyn J, Intille S. Estimating activity and sedentary behavior fron an accelerometer on the hip or wrist. Med Sci Sports Exerc. 2013;45(5):964-75.

24. Freedson PS, Melanson E, Sirard J. Calibration of the Computer Science and Applications, Inc. accelerometer. Med Sci Sports Exerc. 1998;30(5):777-81.

25. Broeck JV, Cunningham SA, Eeckels R, Herbst K. Data cleaning: detecting, diagnosing, and editing data abnormalities. PLoS Med. 2005;2(10):966-70.

26. Ministério da Educação do Brasil. Coordenação de Aperfeiçoamento de Pessoal de Nível Superior. Tabela de áreas de conhecimento/avaliação. Brasília: Ministério da Educação do Brasil; 2017.

27. Bland JM, Altman DG. Statistical methods for assessing agreement between two methods of clinical measurement. Lancet. 1986;1(8476):307-10.

28. Mukaka MM. Statistics Corner: A guide to appropriate use of correlation coefficient in medical research. Malawi Med J. 2012;24(3):69-71.

29. Bakker EA, Hartman YAW, Hopman MTE, Hopkins ND, Graves LEF, Dunstan DW et al. Validity and reliability of subjective methods to assess sedentary behaviour in adults: a systematic review and meta-analysis. Int J Behav Nutr Phys Act. 2020;17(1):75.

30. Sousa TF, Farias GS, Franco DC. Self-reported of sedentary behavior by isaq-a questionnaire for application in undergraduate: analysis of reliability and concurrent validity. Rev Bras Ciênc Mov. 2020;28(1):155-61.

Recebido: 06/01/2021 Aprovado: 06/09/2021

\section{Como citar este artigo:}

Franco DC, Farias GS, Pelegrini A, Virtuoso Junior JS, Sousa TF. Validade das medidas do tempo sentado do questionário IPAQ-versão curta em universitários brasileiros. Rev Bras Ativ Fís Saúde. 2021;26:e0223. DOI: 10.12820/rbafs.26e0223 